\title{
Orbitopatía tiroidea: protocolo de manejo basado en revisión de la evidencia
}

\author{
Thyroid orbitopathy: Management protocol based on review of \\ the evidence
}

\author{
Gómez $C^{1}$, Imitola $A^{1}$, Taboada $L^{2}$, Henao $D C^{1}$, Marín $L^{3}$, Camargo ${ }^{4}$, Kattath $L^{5}$, Fonseca $C^{6}$, \\ Colón $C^{5}$, Ascnher $P^{1}$.
}

${ }^{1}$ Médico Internista Endocrinólogo, Hospital Universitario San Ignacio, Bogotá, Colombia.

${ }^{2}$ Médico Internista, Fellow Endocrinología, Pontificia Universidad Javeriana, Bogotá, Colombia.

${ }^{3}$ Médico Internista Endocrinólogo, Centro Médico "Carlos Ardila Lulle" - Endocrinólogos del Oriente ENDORIENTE, FOSCAL Internacional, Floridablanca, Santander.

${ }^{4}$ Médico Oftalmólogo, Hospital Universitario San Ignacio, Bogotá, Colombia.

${ }^{5}$ Médico Internista, Endocrinólogo, Fundación Santa Fe, Bogotá, Colombia.

${ }^{6}$ Médico Internista, Endocrinólogo.

Autor de correspondencia: Claudia Milena Gómez Giraldo Correo electrónico: cmgg77@hotmail.com

Fecha de recepción: 09/08/2018

Fecha de aceptación: 18/04/2019

\section{Resumen}

La orbitopatía tiroidea es una patología potencialmente grave que amenaza la visión. Es la manifestación extratiroidea más frecuente de la enfermedad de Graves y, aunque la prevalencia no está evaluada en Colombia, puede producir pérdida de la visión, con consecuencias catastróficas. Teniendo en cuenta lo anterior, se realizó este trabajo con el objetivo de describir su enfoque diagnóstico y terapéutico basado en la mejor evidencia disponible.

Se elaboró un protocolo de manejo aplicable a nuestro medio y se realizó una evaluación y adaptación de la guía European Group on Graves' Orbitopathy (EUGOGO) del $2016^{(1)}$. Para la evaluación se usó la herramienta AGREE II y se utilizó la metodología GRADE para la graduación de la calidad de la evidencia y formulación de las recomendaciones ${ }^{(2)}$. Adicionalmente, se hizo una revisión sistemática de la literatura con el objetivo de evaluar la eficacia de la terapia biológica en el manejo de la orbitopatía de Graves.
Se presentan las recomendaciones para el diagnóstico, tratamiento y seguimiento. Se recalca la importancia de contar con un equipo multidisciplinario con experiencia en el manejo de la orbitopatía tiroidea (endocrinología, oftalmología, cirugía de órbita, radioterapia y reumatología), idealmente en un centro de tercer nivel de atención. Es esencial la clasificación adecuada para determinar qué pacientes se benefician del tratamiento inmunosupresor. Si se maneja de forma oportuna, se puede lograr una disminución de la aparición de secuelas irreversibles.

Palabras clave: oftalmopatía de Graves, enfermedad de Graves, glucocorticoides, rituximab, radioterapia.

\section{Abstract}

Thyroid orbitopathy is a potentially serious condition that may threaten vision. It is the most frequent extrathyroidal manifestation of Graves' disease and although the prevalence is not yet evaluated in our country, this complication can produce vision loss with catastrophic consequences. Taking this into account, this work was done with the objective of describing its diagnostic and therapeutic approach, based on the best available evidence.

A management protocol applicable in our context was developed. For this, an evaluation and adaptation of the European Group on Graves' Orbitopathy (EUGOGO) 2016 guideline was done ${ }^{(1)}$. For the evaluation the AGREE II tool was used ${ }^{(2)}$. The GRADE methodology was used to grade the quality of evidence and to formulate recommendations. In addition, a systematic review of the literature was conducted with the objective of evaluating the efficacy of biological therapy in the management of Graves' orbitopathy.

Throughout the document, recommendations for adequate diagnosis, treatment and follow-up are presented. The importance of having a multidisciplinary team with experience in the management of thyroid orbitopathy (endocrinology, ophthal- 
mology, orbit surgery, radiotherapy and rheumatology) is emphasized, ideally in a third level care center. Adequate classification is essential to determine which patients will benefit from immunosuppressive therapy. If it is managed in a timely manner, a decrease in the appearance of irreversible sequelae can be achieved.

Keywords: Graves ophthalmopathy; Graves' disease; glucocorticoids; rituximab; radiotherapy.

\section{Introducción}

La orbitopatía tiroidea es una patología potencialmente grave que amenaza la visión. Es la manifestación extratiroidea más frecuente de la enfermedad de Graves y se caracteriza por una fase inicial de actividad inflamatoria, que puede tardar de 1 a 3 años en resolverse (fase activa), seguida de una fase de fibrosis y cicatrización (fase inactiva) ${ }^{(3)}$. El $5 \%$ de los casos puede presentarse en pacientes eutiroideos o con hipotiroidismo autoinmune ${ }^{(4)}$. Afecta entre el $25 \%$ y $50 \%$ de los pacientes con enfermedad de Graves, con compromiso severo hasta en el 5\%. La orbitopatía tiroidea usualmente ocurre de forma simultánea con el debut del hipertiroidismo o con una diferencia de 18 meses, y menos frecuentemente puede preceder o debutar años después. Usualmente el compromiso es bilateral ${ }^{(4,5)}$.

Aunque la prevalencia no está evaluada en nuestro país, esta complicación puede producir pérdida de la visión, con consecuencias catastróficas. El objetivo de este documento es presentar un protocolo de manejo adaptado de las guías EUGOGO 2016, en el que se incluye, además, una revisión de la evidencia disponible para cada recomendación. De esta manera se busca difundir los conocimientos básicos entre los especialistas que tratan esta patología con el fin de reducir la limitación funcional y la alteración en la calidad de vida.

Teniendo en cuenta la posibilidad de falla a los tratamientos de primera línea y la aparición de nuevas posibilidades de tratamiento no incluidas en las guías, se realizó una revisión sistemática de la literatura con el fin de evaluar la eficacia de la terapia biológica en el manejo de la orbitopatía de Graves.

\section{Metodología}

Se realizó una adaptación de la guía EUGOGO 2016(1). Se aplicó el instrumento para la evaluación de guías de práctica clínica AGREE II a la guía, con el fin de determinar su calidad y rigor metodológico ${ }^{(2)}$. La evaluación fue llevada a cabo por 2 sujetos. Se formularon las recomendaciones y se expresó la calidad de la evidencia utilizando la metodología GRADE, con el siguiente sistema de codificación:

- Se indica recomendación fuerte y se asocia con la frase "nosotros recomendamos"

- Denota recomendación débil y se asocia con la frase "nosotros sugerimos"
La calidad de la evidencia se codificó así:

- $\quad$ Muy baja (+)

- $\quad$ Baja $(++)$

- Moderada $(+++)$

- $\quad$ Alta $(++++)$.

Para la evaluación de la eficacia de la terapia biológica como parte del tratamiento de la enfermedad de Graves se realizó una revisión sistemática de la literatura. Un autor efectuó la búsqueda en Pubmed, Embase y Cochrane Central Register of Controlled Trials. Se utilizaron los siguientes términos de búsqueda: Graves' orbitopathy, Graves' ophthalmopathy, rituximab, tocilizumab y teprotumumab y se utilizaron operadores booleanos $A N D$ y $O R$ para construir las estrategias de búsqueda propias a cada medicamento.

Se incluyeron estudios clínicos aleatorizados, sin embargo, en los casos en los que no se encontraran este tipo de estudios, se incluyeron estudios observacionales prospectivos. Para delimitar el tipo de estudio, se utilizaron los filtros respectivos en cada base de datos. Se incluyeron estudios en inglés o en español sin restricción de fecha hasta el momento de la búsqueda (noviembre de 2018) y que describieran la eficacia con respecto a desenlaces clínicos. Se excluyeron los reportes de caso. Los resultados de la estrategia de búsqueda y los resultados de los estudios incluidos se describirán dentro del protocolo de manejo presentado.

\section{Resultados}

Protocolo de manejo de pacientes con orbitopatía tiroidea

Se obtuvo una puntuación global de $74 \%$ como resultado de la evaluación de la guía EUGOGO 2016 con la herramienta AGREE II. En los dominios 4 y 6 se obtuvo un alto porcentaje, $100 \%$ y $83 \%$, respectivamente. Se concluyó que es una guía adecuada para emitir recomendaciones, con la debilidad de no contar con población latina.

\section{Tratamiento de la enfermedad tiroidea en pacientes con orbitopatía tiroidea}

Para pacientes con orbitopatía tiroidea activa modera/severa o con amenaza de pérdida de la visión se recomienda que el tratamiento del hipertiroidismo se realice con antitiroideos o con tiroidectomía y que la función tiroidea se mantenga de manera estable $(1,++++)$. En un metaanálisis que incluyó a 1404 participantes que habían recibido distintas opciones de tratamiento para la enfermedad de Graves se encontró que la terapia con yodo, comparada con los antitiroideos y la cirugía, aumenta significativamente la incidencia de nueva presentación o deterioro de la orbitopatía tiroidea (OR 2,958; IC 95\% 1,731-4,735 de terapia con yodo frente a antitiroideos; y OR 3,205; IC 95\% 1,053-6,88 de terapia con yodo vs. cirugía). La 
diferencia entre antitiroideos y la cirugía no fue significativa (OR 1,123; IC 95\% 0,3583-2,389) (6).

En pacientes con orbitopatía inactiva, levemente activa y sin factores de riesgo de presentación de novo o deterioro de la orbitopatía tiroidea, como tabaquismo, niveles de T3 altos ( $\geq 325 \mathrm{ng} / \mathrm{dL}$ o $\geq 5 \mathrm{nmol} / \mathrm{L}$ ) o títulos elevados de anticuerpos antirreceptores de TSH (TRAb) (>50\% de inhibición o >8,8 UI/L), podría considerarse la terapia con yodo para el manejo del hipertiroidismo ${ }^{(3)}$. En pacientes con orbitopatía tiroidea activa leve sin contraindicación para el uso de glucocorticoides y que vayan a ser llevados a terapia con yodo se recomienda la administración de esteroides para disminuir el riesgo de progresión. El tratamiento con prednisona en dosis de 0,3 a $0,5 \mathrm{mg} / \mathrm{kg} /$ día se recomienda para pacientes con múltiples factores de riesgo o de $0,2 \mathrm{mg} / \mathrm{kg} /$ día para pacientes de bajo riesgo, iniciado de forma concomitante con la yodoterapia y por un período de 3 meses. Los pacientes con orbitopatía inactiva pueden recibir el yodo radiactivo sin cobertura con esteroides y evitar, en lo posible, la aparición de hipotiroidismo posyodo y tabaquismo $(1,++++)^{(1)}$.

\section{Factores de riesgo para el desarrollo o el deterioro de la orbitopatía tiroidea}

El hipertiroidismo y el hipotiroidismo son deletéreos en el curso clínico de esta enfermedad, por lo que se recomienda restaurar y mantener la normalidad de la función tiroidea tan pronto como sea posible $(1,++++)^{(7,8)}$.

La terapia con yodo radioactivo está contraindicada en orbitopatía tiroidea activa moderada/severa o con amenaza de pérdida de la visión por el riesgo de deterioro del compromiso orbitario $(1,++++)$. El yodo radioactivo confiere un riesgo definido de exacerbación o aparición de orbitopatía, particularmente en fumadores, hipertiroidismo severo, altos niveles de TRAb e hipertiroidismo de reciente inicio ${ }^{(7-12)}$. Múltiples estudios han identificado que el riesgo de progresión de la orbitopatía tiroidea luego de la terapia con yodo oscila entre $10 \%$ a $39 \%{ }^{(13)}$. La asociación entre el cigarrillo y la orbitopatía tiroidea es ampliamente conocida, por lo cual es prioritario el cese del tabaquismo $(1,++++)^{(14)}$.

\section{Evaluación de la actividad y severidad de la enfermedad orbitaria}

La selección del mejor tratamiento depende de la actividad y de la severidad de la orbitopatía. Se recomienda que la actividad sea evaluada de acuerdo con los criterios estandarizados por la puntuación de actividad clínica (CAS), como activa e inactiva $(1,+++)^{(1)}$. Del CAS existen 2 versiones: una de 7 ítems y otra de 10 ítems ${ }^{(1)}$. En cada consulta de endocrinología se recomienda realizar el CAS de 7 ítems (tabla 1). Un CAS $\geq 3 / 7$ es indicativo de actividad. Cuando la actividad es evaluada por oftalmología se recomienda realizar la puntuación de CAS de 7 ítems en la consulta inicial, pero para el seguimiento se aconseja el CAS de 10 ítems (tabla 2), con el cual se puede evaluar la presencia de miopatía, que es indicativa de progresión de la enfermedad ${ }^{(15)}$.

Tabla 1. Evaluación inicial de la actividad de la orbitopatía tiroidea según CAS ${ }^{(1)}$

\begin{tabular}{l|c}
\multicolumn{1}{|c|}{ Ítem a evaluar } & Puntos \\
\hline Dolor retrobulbar espontáneo & 1 \\
\hline $\begin{array}{l}\text { Dolor con los movimientos de mirada hacia } \\
\text { arriba y hacia abajo }\end{array}$ & 1 \\
\hline Enrojecimiento palpebral & 1 \\
\hline Enrojecimiento conjuntival & 1 \\
\hline Edema de la carúncula & 1 \\
\hline Edema palpebral & 1 \\
\hline Edema conjuntival & 1 \\
\hline Total de puntos & 7 \\
\hline Orbitopatía activa: $\geq 3 / 7$ puntos & \\
\hline Orbitopatía inactiva: $<3 / 7$ puntos & \\
\hline
\end{tabular}

Tabla 2. Evaluación de seguimiento de la actividad de la orbitopatía tiroidea según CAS ${ }^{(15)}$

Ítem a evaluar

Puntos

\begin{tabular}{l|c}
\hline Dolor retrobulbar espontáneo & 1 \\
\hline $\begin{array}{l}\text { Dolor con los movimientos de mirada hacia arriba } \\
\text { y hacia abajo }\end{array}$ & 1 \\
\hline Enrojecimiento palpebral & 1 \\
\hline Enrojecimiento conjuntival & 1 \\
\hline Edema de la carúncula & 1 \\
\hline Edema palpebral & 1 \\
\hline Edema conjuntival & 1 \\
\hline $\begin{array}{l}\text { Incremento de la proptosis } \geq 2 \mathrm{~mm} \text { durante un } \\
\text { período de } 1 \text { a } 3 \text { meses }\end{array}$ & 1 \\
\hline
\end{tabular}

Disminución de los movimientos oculares en cualquier dirección $\geq 8^{\circ}$ durante un período de 1 a 3 meses

1

Disminución de la agudeza visual $\geq 1$ línea en la tabla de Snellen durante un período de 1 a 3 meses

Total de puntos

Orbitopatía activa: $\geq 4 / 10$ puntos

Orbitopatía inactiva: $<4 / 10$ puntos

Se recomienda que la severidad sea evaluada por oftalmología en cada consulta de acuerdo con los criterios estandarizados por EUGOGO (tabla 3), clasificando la orbitopatía como leve, moderada a severa o con amenaza de pérdida de la visión $(1,+++)^{(1)}$. 
Tabla 3. Evaluación de la severidad de la enfermedad orbitaria según EUGOGO ${ }^{(1)}$

Moderada/severa
Dos o más de los siguientes:
- Retracción palpebral $\geq 2 \mathrm{~mm}$
- Afectación de las partes blandas moderada o grave
- Exoftalmos $\geq 3 \mathrm{~mm}$ por encima de lo normal para raza y
género
- Diplopía inconstante o constante

Amenaza de pérdida de la visión

- Neuropatía óptica distiroidea

- Ulceración y exposición corneal

- Subluxación del globo ocular

- Formas severas de globo congelado

- Pliegues coroideos

\section{Estudios complementarios}

El diagnóstico de la orbitopatía tiroidea casi siempre es clínico, sin embargo, hasta en $15 \%$ de los pacientes la presentación puede ser asimétrica o unilateral, siendo un verdadero reto. En este caso, las imágenes permiten realizar un diagnóstico diferencial. La resonancia nuclear magnética, la tomografía computarizada y la ecografía de órbitas permiten evaluar el tamaño de los músculos extraoculares, el volumen del tejido graso orbitario y las glándulas lagrimales.

La tomografía de órbitas es la imagen de elección en la planeación de la descompresión quirúrgica. Dentro de los hallazgos que pueden encontrarse están el exoftalmos, el engrosamiento de vientres musculares, el incremento en el volumen de la grasa y, en especial, permite la evaluación precisa del ápex, con lo que se puede documentar la presencia de compresión del nervio óptico en el vértice orbitario $(1,+++)^{(16-18)}$.

En manos experimentadas, la ecografía de órbitas con Doppler color permite monitorizar la respuesta al tratamiento. Sus desventajas incluyen la variabilidad interobservador, reproducibilidad y limitación para la evaluación del ápex y del músculo recto-inferior, que es el más frecuentemente comprometido en la orbitopatía de Graves, siendo su precisión anatómica menor cuando se compara con otras técnicas diagnósticas ${ }^{(18,19)}$.

La resonancia, aunque tiene mayor sensibilidad en fases inactivas de la enfermedad, no brinda mayores ventajas frente al TAC. Tiene limitaciones para la caracterización de estructuras óseas y se debe tener en cuenta su mayor costo y menor disponibilidad ${ }^{(18)}$.

Estos estudios se encuentran indicados en pacientes con proptosis ocular unilateral o de etiología no clara, sospecha de neuropatía óptica y previo a cirugía descompresiva de órbita $(1,+++)^{(16)}$.

En el contexto de la orbitopatía tiroidea, los TRAb tienen las siguientes utilidades: realizar diagnóstico en pacientes eutiroi- deos, hipotiroideos o con orbitopatía unilateral, determinar el pronóstico de estadios tempranos, correlación con neuropatía óptica distiroidea y como marcador de progresión ${ }^{(19-23)}$.

\section{Tratamiento de la orbitopatía tiroidea}

Se recomienda que todos los pacientes se traten con lágrimas artificiales sin conservantes y con propiedades osmoprotectivas, a menos que exista exposición de la córnea que requiera mayor protección con geles o ungüentos especialmente para uso nocturno $(1,++)$. Se recomienda el uso de gafas protectoras, dormir con la cabecera elevada, uso de cámara húmeda en las noches y prismas de Fresnel adaptados en las gafas de uso diario ${ }^{(24)}$. Para disminuir la retracción palpebral, se puede aplicar toxina botulínica $(1,++)^{(25)}$.

\section{Tratamiento de la enfermedad orbitaria leve activa}

En pacientes con orbitopatía tiroidea leve activa de menos de 18 meses de duración, se recomienda dar suplencia con selenio (Se) en dosis de $100 \mu$ g cada 12 horas por vía oral por 6 meses $(1,+++)^{(1)}$. En un estudio comparado con pentoxifilina y placebo se mejoró la calidad de vida y la evaluación ocular global a los 6 meses del tratamiento. La tasa de progresión a estados más severos fue menor con Se $(p<0,001)^{(19,26)}$. Los hallazgos de este estudio han llevado a considerar su uso en orbitopatía tiroidea leve activa, pero hay que tener en cuenta que el estudio se realizó en una población europea que habita regiones con déficit de Se y aún es incierto el beneficio en pacientes provenientes de áreas con suficiencias de Se.

\section{Tratamiento de la enfermedad orbitaria moderada/ severa activa}

\section{Manejo de primera línea}

\section{Glucocorticoides intravenosos (IV)}

Para la mayoría de los casos se recomienda metilprednisolona en dosis acumulada de 4,5 g, divididos en aplicaciones durante 12 semanas. Las primeras 6 semanas $500 \mathrm{mg}$ semanal, seguidos de $250 \mathrm{mg}$ semanal por 6 semanas más. En formas más severas se puede utilizar 7,5 gramos de dosis acumulada, iniciando con dosis de $750 \mathrm{mg}$ semanal por 6 semanas, seguidos de 500 mg semanal por 6 semanas más $(1,++++)^{(1)}$.

Distintos estudios han demostrado mejores tasas de respuesta en pacientes tratados con glucocorticoides IV en comparación con pacientes tratados con glucocorticoides orales y se documentan tasas de respuesta entre el $63 \%$ a $77 \%$, con menor número de reacciones adversas (39\% para IV y $81 \%$ para orales, $p<0,001)^{(1,18,27-30)}$.

En un metaanálisis que tenía como objetivo comparar diferentes monoterapias (glucocorticoides orales, glucocorticoides IV, radioterapia orbitaria o inyecciones retrobulbares de glucocorticoides) en pacientes con orbitopatía moderada/ 
severa activa, los glucocorticoides IV mostraron una tasa de respuesta superior a los orales (RR 1,48; IC 95\% 1,18-1,87, $p$ $=0,0007$ ). Los glucocorticoides retrobulbares no demostraron diferencias con los orales y no hubo diferencias entre los orales vs. radioterapia. Los glucocorticoides IV, la radioterapia y las inyecciones retrobulbares presentaron una menor tasa de eventos adversos frente a los glucocorticoides orales ${ }^{(31)}$. Otro metaanálisis evaluó 4 estudios que comparaban glucocorticoides IV frente a orales en orbitopatía activa y se encontró superioridad de los glucocorticoides IV sobre los orales en reducción del CAS, especialmente en orbitopatía severa (CAS $>5$ ), con mayores eventos adversos en pacientes con glucocorticoides orales ${ }^{(28)}$.

La dosis recomendada para la mayoría de los pacientes con orbitopatía activa moderada de 4,5 g en 12 semanas está respaldada por un estudio que comparó la metilprednisolona IV con la prednisolona oral, donde se demostró mayor efectividad y mejor tolerancia con el régimen IV ${ }^{(32)}$. Otro estudio comparó este esquema de tratamiento con un régimen más corto con igual dosis acumulada (infusión diaria de $500 \mathrm{mg}$ por 3 días por 2 semanas, seguido de una infusión diaria de $250 \mathrm{mg}$ por 3 días por 2 semanas más), sin embargo, el esquema corto se asoció con menor eficacia que el protocolo estándar (tasa de respuesta de $41 \%$ frente a $77 \%, p=0,0025$ ) y con mayor toxicidad ${ }^{(33)}$. En formas más severas se puede utilizar dosis acumulada de 7,5 g de metilprednisolona ${ }^{(34)}$. Esta recomendación parte de un estudio en pacientes con orbitopatía activa modera/severa, los cuales recibieron 3 regímenes de dosis acumulada de metilprednisolona $(7,47,4,98$ y 2,25 g) en el mismo período. Hubo mejoría oftálmica con todas las dosis, pero fue más alta en los que recibieron mayor dosis ( $52 \%$ vs. $35 \%$ y $28 \%$, respectivamente), sin embargo, este beneficio fue transitorio y se asoció con mayor toxicidad con la dosis más alta, por tanto, no se considera de elección para la mayoría de los pacientes ${ }^{(34)}$.

Las reacciones adversas se han relacionado con enfermedades preexistentes o con los esquemas de glucocorticoides utilizados. Dosis de metilprednisolona mayores de 0,5 gramos varias veces por semana por 2 semanas consecutivas deben reservarse para pacientes con orbitopatía tiroidea activa con amenaza de pérdida de visión, ya que estos protocolos o dosis acumuladas superiores a 8 gramos se asocian con mayores tasas de reacciones adversas ${ }^{(27)}$.

En una revisión que incluyó a más de 1000 pacientes tratados con glucocorticoides IV se encontró que la morbilidad y mortalidad de los glucocorticoides IV fue de 6,5\% y 0,6\%, respectivamente. Los eventos adversos graves ocurrieron en pacientes que recibieron dosis diarias o alternas mayores de $0,5 \mathrm{~g}$ ( 5 pacientes), de los cuales 2 murieron por complicaciones cardiovasculares y cerebrovasculares ${ }^{(27)}$. Datos de diferentes estudios reportan falla hepática severa después de los pulsos IV de glucocorticoides, con insuficiencia hepática aguda mortal en 4 pacientes con orbitopatía tiroidea, con dosis acumuladas entre 10-24 g ${ }^{(27)}$.
La tolerabilidad hepática es mejor cuando los bolos son aplicados semanalmente. La hepatitis B preexistente es un factor de riesgo para presentar el daño hepático. Antes de iniciar el tratamiento se deben medir las enzimas hepáticas, los marcadores virales para hepatitis, la glucosa plasmática en ayunas y algunos recomiendan realizar ecografía hepática ${ }^{(1)}$. El uso concomitante de estatinas puede potencializar el daño hepático, por lo cual se sugiere una suspensión temporal en caso de que la situación del paciente lo permita ${ }^{(15)}$. Además, en nuestro medio recomendamos desparasitar previo a la administración de metilprednisolona.

Las contraindicaciones a la terapia incluyen hepatitis viral reciente, disfunción hepática significativa, morbilidad cardiovascular severa, hipertensión arterial no controlada, trastornos psiquiátricos y diabetes mellitus mal controlada ${ }^{(1)}$. Durante el tratamiento se recomienda monitorizar las enzimas hepáticas, los niveles de glucosa y la presión arterial. Se deben utilizar inhibidores de bomba de protones y protección ósea, especialmente en pacientes con múltiples factores de riesgo para osteoporosis y en todo momento hacer un balance entre el riesgo y el beneficio, considerando otras opciones de manejo si en algún instante el riesgo excede el beneficio ${ }^{(1)}$.

En Colombia, Vargas y Maya publicaron en 2013 una revisión sistemática de ensayos clínicos controlados desde enero de 1980, con el objetivo de evaluar el efecto de la administración de un glucocorticoide IV comparado con un glucocorticoide oral, sus posibles efectos adversos y esquemas de tratamiento. Los autores documentaron 4 ensayos clínicos publicados desde 2005, 3 de los cuales demostraron diferencias estadísticamente significativas en la mayoría de los parámetros evaluados a favor de los que recibieron el esteroide por vía IV, los cuales también tuvieron una mejoría más temprana. No se encontraron efectos adversos serios, pero en los pacientes con esteroide oral $(p<0,0001)$ hubo mayor ganancia de peso, disminución de la densidad mineral ósea (DMO) en la columna lumbar, depresión, hipertensión arterial y neuropatía óptica. Los datos son escasos en relación con el uso de esteroides IV diferentes a la metilprednisolona, por ejemplo, dexametasona. Los pacientes que presentaron mayor beneficio fueron los clasificados con orbitopatía tiroidea moderada o severa, con un puntaje en escala de actividad $>4$. El esquema de aplicación de metilprednisolona IV según 3 de los 4 estudios revisados (y probablemente el más aceptado) es de 0,5 g cada semana por 6 semanas, luego $0,25 \mathrm{~g}$ cada semana por 6 semanas, con una dosis acumulada de 4,5 gramos en 12 semanas ${ }^{(35)}$.

\section{Manejo de segunda línea}

Se considera su uso cuando no hay respuesta al tratamiento, definida como persistencia de actividad según CAS o cuando hay recaída tras los bolos de metilprednisolona, lo cual puede presentarse hasta en $30 \%$ de los casos, lo que impone un desafío para el manejo de esta patología dada la evidencia 
limitada sobre la eficacia de los regímenes alternativos; por tanto, se recomienda que la decisión sea compartida entre el sujeto, el oftalmólogo y el endocrinólogo, evaluando cada opción en el contexto del paciente y teniendo en cuenta sus comorbilidades, circunstancias personales e impacto en la calidad de vida $(1,++)$.

Se pueden considerar las siguientes opciones:

\section{Repetir bolos de metilprednisolona IV}

En caso de que el paciente tolere la terapia y que no exceda la dosis acumulada de 8 gramos de metilprednisolona ${ }^{(1)}$.

\section{Radioterapia combinada con esteroides sistémicos}

Se ha descrito el rol de la radioterapia en la orbitopatía tiroidea activa moderada/severa por su efecto inmunosupresor local, especialmente en estadios tempranos de la enfermedad, con menos de 1 año de evolución ${ }^{(36)}$. La combinación de glucocorticoides orales más radioterapia es más efectiva que los glucocorticoides orales en inactivación de la orbitopatía y se han reportado desenlaces favorables en el $60 \%$ a $85 \%$ de los pacientes ${ }^{(37)}$.

En un metaanálisis se comparó la combinación de esteroide sistémicos y radioterapia orbital vs. esteroide sistémico solo o radioterapia sola y se encontró mayor efectividad para la terapia combinada ${ }^{(28)}$. No se dispone de evidencia suficiente para recomendar un esquema específico de tratamiento, pero las guías recomiendan que la radioterapia se administre con glucocorticoide oral debido a que la evidencia de mayor calidad existe para esta combinación, aunque hay una posibilidad de utilizarla con glucocorticoides IV ${ }^{(1,38,39)}$. La radioterapia orbitaria puede considerarse como terapia de segunda línea en pacientes con intolerancia a glucocorticoides o dosis acumulada mayor de $8 \mathrm{~g}$ de metilprednisolona IV, control inadecuado de síntomas inflamatorios e imposibilidad del retiro de los glucocorticoides ${ }^{(36)}$.

Por lo general, es una terapia segura a corto y a largo plazo. A corto plazo puede producir eritema local y alopecia temporal. A largo plazo se puede presentar retinopatía microvascular en $1 \%$ a $2 \%$ de los casos y desarrollo de neoplasias radio inducidas (extremadamente raro) ${ }^{(36)}$. Por las razones previamente descritas, la radioterapia orbitaria está contraindicada en pacientes con retinopatía diabética e hipertensiva severa y debe evitarse en pacientes jóvenes menores de 35 años. Se ha descrito también mayor prevalencia de catarata.

\section{Terapia biológica}

\section{Tocilizumab}

Se encontraron 15 estudios tras realizar la estrategia de búsqueda, con un solo estudio clínico aleatorizado. En un estudio prospectivo sin grupo control de 18 pacientes con orbitopatía tiroidea activa refractaria a esteroides con CAS $\geq 4$ e inmunoglobulina estimulante tiroidea (TSI) $>10 \mathrm{UI} / \mathrm{mL}$, se administró tocilizumab $8 \mathrm{mg} / \mathrm{kg}$ cada 4 semanas por 4 ciclos o hasta lograr CAS $<1$ o TSI negativo. Se encontró una reducción de la proptosis ocular en $72,2 \%$ de los casos, mejoría en los movimientos oculares en $83,3 \%$ de los casos y resolución de la diplopía en 53,8\% de los pacientes. La disminución de la puntuación del CAS fue estadísticamente significativa $(5,89 \pm 1,41$; $p<0,00027$ ) durante el seguimiento a 9 meses ${ }^{(40)}$.

En el ensayo clínico aleatorizado publicado en 2018 se incluyeron a 32 pacientes con orbitopatía tiroidea activa moderada-severa resistente a esteroides que recibieron tocilizumab $8 \mathrm{mg} / \mathrm{kg}$ de peso o placebo, IV, a las semanas 0, 4, 8 y 12 y fueron seguidos durante 28 semanas adicionales. Se encontró una reducción de al menos 2 puntos en el CAS desde la basal a la semana 16 en el 93,3\% de los pacientes que recibieron tocilizumab frente a $58,8 \%$ de los que recibieron placebo $(p$ $=0,04)$. También se encontró una diferencia significativa en la proporción de pacientes que alcanzaron un CAS $<3(86,7 \%$ frente a $35,2 \%, p=0,005$ ) entre los pacientes que recibieron tocilizumab y placebo, respectivamente ${ }^{(41)}$.

\section{Rituximab}

Tras realizar la búsqueda se obtuvieron 8 resultados, de los cuales 4 correspondían a estudios clínicos aleatorizados, sin embargo, uno de ellos se excluyó debido a que la intervención consistía en rituximab en combinación con yodo radioactivo y otro de ellos se excluyó por no brindar información de desenlaces clínicos. Se revisaron entonces dos estudios clínicos aleatorizados. El primero comparó rituximab vs. metilprednisolona con dosis acumulada de 7,5 g en pacientes con orbitopatía activa moderada/severa. Se reportó mayor inactivación de la enfermedad con rituximab comparado con metilprednisolona (100\% vs. $69 \%$ ) a las 24 semanas y mayor reducción de signos de actividad con rituximab. Por otro lado, los efectos adversos fueron más frecuentes con rituximab (86\%) que con metilprednisolona (62\%). Dos de las reacciones con rituximab se consideraron mayores y se relacionaron con la infusión ${ }^{(42)}$.

El segundo estudio evaluó los efectos de rituximab comparado con placebo en 25 pacientes con orbitopatía tiroidea con CAS $\geq 4$ que hubiesen tenido signos y síntomas de progresión en los 2 meses previos o que no hubieran tenido mejoría con el tratamiento en los 6 meses previos. El rituximab no demostró beneficios respecto al placebo y se plantea un posible impacto de la cronicidad de la enfermedad en el resultado ${ }^{(43)}$. Se necesitan ensayos clínicos aleatorizados controlados de mayor tamaño que puedan generar una recomendación clara respecto al uso de este medicamento. Por el momento recomendamos que se puede considerar como tratamiento de segunda línea en pacientes que no han respondido con glucocorticoide IV y que no han tenido una enfermedad prolongada (más de 6 meses), pero se debe tener en cuenta los efectos adversos que pudieran derivarse de este manejo. 


\section{Teprotumumab}

Tras realizar la búsqueda se obtuvieron 8 resultados, sin embargo, todos correspondían a artículos de revisión, excepto un resultado que pertenecía a un estudio clínico aleatorizado controlado con placebo que incluyó 88 sujetos, en el que se evaluó la eficacia y la efectividad de teprotumumab en pacientes con orbitopatía tiroidea activa moderada/severa de menos de 9 meses de evolución, sin antecedente de tratamiento quirúrgico o de tratamiento médico (excepto esteroide oral con dosis acumulada menor de $1 \mathrm{~g}$ de metilprednisolona o su equivalente ${ }^{(44)}$. Teprotumumab se asoció con reducción de 20 más puntos del CAS en el $69 \%$ de los pacientes comparado con $20 \%$ en el grupo placebo (OR ajustado 8,$86 ; p<0,001$ ) y reducción en $4 \mathrm{~mm}$ o más en la proptosis en el $40 \%$ de los pacientes frente a $0 \%$ en el grupo placebo. La hiperglucemia fue el efecto adverso más relevante que se presentó en pacientes con antecedente de diabetes mellitus. Con los resultados de este estudio, en agosto de 2016 el teprotumumab fue designado como medicamento huérfano por la FDA para esta patología $(1,+++)$. Aún no se encuentra disponible en nuestro país.

\section{Ciclosporina}

Acompañada de glucocorticoides orales, la ciclosporina se recomienda como terapia de segunda línea para el tratamiento de la orbitopatía tiroidea. Su respuesta es mejor cuando se administra en la combinación descrita que cuando se utiliza sola ${ }^{(1,45)}$.

\section{Tratamiento de los casos de amenaza de pérdida de la visión \\ Neuropatía óptica distiroidea}

Es una emergencia que requiere tratamiento inmediato, el cual debe hacerse con metilprednisolona en dosis de $1 \mathrm{~g}$ IV cada día por 3 días consecutivos. El paciente se debe evaluar a la semana y, si no hay mejoría, se recomienda realizar descompresión orbitaria de urgencia. Si el paciente presenta respuesta parcial se recomienda repetir los bolos y reevaluar a la semana y si tiene respuesta completa se aconseja llevar a dosis total de metilprednisolona de 7,5 g. En caso de que exista un deterioro rápido en la función visual se recomienda realizar una cirugía descompresiva de urgencia $(1,+++)^{(1)}$.

\section{Ulceración y exposición corneal}

Se recomienda que la exposición corneal severa sea tratada con metilprednisolona IV o con cirugía descompresiva de urgencia tan pronto como sea posible con el fin de evitar la progresión a la ruptura de la córnea. En caso de que se presente esta última debe tratarse de inmediato quirúrgicamente $(1,+++)^{(1)}$. Se recomienda que las formas de subluxación del globo ocular y pliegues coroideos de reciente aparición sean tratados con cirugía descompresiva de urgencia tan pronto como sea posible $(1,+++)^{(1)}$.

\section{Tratamiento de la enfermedad orbitaria inactiva}

Requiere diferentes grados de rehabilitación quirúrgica, la cual es liderada por el servicio de oftalmología. El tipo de intervención depende del deterioro funcional o estético que persista en la fase postinflamatoria de la enfermedad y su impacto en la calidad de vida.

\section{Complejidad y sitio de la atención}

Se recomienda que los pacientes sean referidos al servicio de urgencias de un hospital de tercer nivel que cuente como mínimo con un endocrinólogo y oftalmólogo con experiencia en el tema, con excepción de los casos más leves con mejoría rápida de la función tiroidea, que pueden ser manejados con lubricantes locales $(1,++)$.

\section{Conclusiones}

La orbitopatía tiroidea es la manifestación más frecuente de la enfermedad de Graves, la cual tiene un impacto negativo en la calidad de vida. Es importante hacer el diagnóstico a tiempo para iniciar el tratamiento oportunamente y disminuir las consecuencias catastróficas.

Para todos los pacientes se debe recalcar la necesidad de evitar el tabaquismo y restaurar cuanto antes la función tiroidea. Para los pacientes con enfermedad activa leve se les debe dar la oportunidad de recibir manejo tópico, además del uso de antioxidantes como Se. Para los pacientes con orbitopatía tiroidea activa moderada a severa se deben utilizar bolos de metilprednisolona IV y, según la respuesta, evaluar la necesidad de otras terapias de rescate, en las cuales la evidencia clínica es baja. Para los pacientes con orbitopatía tiroidea activa con amenaza de pérdida de la visión, la metilprednisolona IV en dosis alta es la primera línea de tratamiento, pero se debe considerar la descompresión quirúrgica cuando no haya respuesta o si durante la terapia existe un deterioro rápido en la función visual. Para los pacientes con enfermedad inactiva, las opciones de manejo incluyen el tratamiento tópico y las cirugías de rehabilitación.

\section{Declaración de fuentes de financiación y posibles conflic-} tos de interés

Fuentes de financiación: recursos propios de la unidad de Endocrinología del Hospital Universitario San Ignacio.

Declaramos que no hay conflicto de interés. 


\section{Referencias}

1. Bartalena L, Baldeschi L, Boboridis K, Eckstein A, Kahaly GJ, Marcocci C, et al. The 2016 European Thyroid Association/European Group on Graves' orbitopathy guidelines for the management of graves' orbitopathy. Eur Thyroid J. 2016;5(1):9-26.

2. Consortium ANS. El Instrumento AGREE II Versión electrónica. 2009. Disponible en: http://www.agreetrust.org.

3. Bahn RS, Burch HB, Cooper DS, Garber JR, Greenlee MC, Klein I, et al. Hyperthyroidism and other causes of thyrotoxicosis: management guidelines of the American Thyroid Association and American Association of Clinical Endocrinologists. Endocr Pract. 2011;17(3):456-520.

4. Bahn RS. Graves' ophthalmopathy. New Engl J. 2010;362:726-38.

5. De Leo S, Lee SY, Braverman LE. Hyperthyroidism. Lancet. 2016;116(8):1477-90.

6. Ren Z, Qin L, Wang JQ, Li Y, Li J, Zhang RG. Comparative efficacy of four treatments in patients with Graves' disease: a network meta-analysis. Exp Clin Endocrinol Diabetes. 2015;123(5):317-22.

7. Tallstedt L, Lundell G, Torring O, Wallin G, Ljunggren JG, Blomgren H, et al. Occurrence of ophthalmopathy after treatment for Graves' hyperthyroidism. The Thyroid Study Group. N Engl J Med. 1992;326(26):1733-8.

8. Acharya SH, Avenell A, Philip S, Burr J, Bevan JS, Abraham P. Radioiodine therapy (RAI) for Graves' disease (GD) and the effect on ophthalmopathy: a systematic review. Clin Endocrinol (Oxf). 2008;69(6):943-50.

9. Eckstein AK, Plicht M, Lax H, Neuhauser M, Mann K, Lederbogen S, et al. Thyrotropin receptor autoantibodies are independent risk factors for Graves' ophthalmopathy and help to predict severity and outcome of the disease. J Clin Endocrinol Metab. 2006;91(9):3464-70.

10. Gerding MN, van der Meer JW, Broenink M, Bakker O, Wiersinga WM, Prummel MF. Association of thyrotrophin receptor antibodies with the clinical features of Graves' ophthalmopathy. Clin Endocrinol (Oxf). 2000;52(3):267-71.

11. Traisk F, Tallstedt L, Abraham-Nordling M, Andersson T, Berg G, Calissendorff J, et al. Thyroid-associated ophthalmopathy after treatment for Graves' hyperthyroidism with antithyroid drugs or iodine-131. J Clin Endocrinol Metab. 2009;94(10):3700-7

12. Vannucchi G, Campi I, Covelli D, Dazzi D, Curro N, Simonetta S, et al. Graves orbitopathy activation after radioactive iodine therapy with and without steroid prophylaxis. J Clin Endocrinol Metab. 2009;94(9):3381-6.

13. Ross DS, Burch HB, Cooper DS, Greenlee MC, Laurberg P, Maia AL, et al 2016 American Thyroid Association guidelines for diagnosis and management of hyperthyroidism and other causes of thyrotoxicosis. Thyroid. 2016;26(10):1343-421.

14. Bartalena L, Martino E, Marcocci C, Bogazzi F, Panicucci M, Velluzzi F, et al More on smoking habits and Graves' ophthalmopathy. J Endocrinol Invest. 1989;12(10):733-7

15. Campi I, Vannucchi G, Salvi M. Therapy of endocrine disease: endocrine dilemma: management of Graves' orbitopathy. Eur J Endocrinol. 2016;175(3):R117-33.

16. Muller-Forell W, Kahaly GJ. Neuroimaging of Graves' orbitopathy. Best Pract Res Clin Endocrinol Metab. 2012;26(3):259-71.

17. McKeag D, Lane C, Lazarus JH, Baldeschi L, Boboridis K, Dickinson AJ, et al. Clinical features of dysthyroid optic neuropathy: a European Group on Graves' Orbitopathy (EUGOGO) survey. Br J Ophthalmol. 2007;91(4):455-8.

18. Vargas-Uricoechea $\mathrm{H}$, Sierra-Torres $\mathrm{CH}$, Meza-Cabrera IA. Tratamiento de la enfermedad de Graves Basedow. Medicina. 2013;35(2):130-49.

19. Khong JJ, McNab A, Ebeling PR, Craig JE, Selva D. Pathogenesis of thyroid eye disease: review and update on molecular mechanisms. Br J Ophthalmol. 2015;100(1):142-50.

20. Vargas-Uricoechea H, Sierra-Torres CH, Meza-Cabrera IA. Enfermedad de Graves-Basedow fisiopatología y diagnóstico. Medicina. 2013;35(1):41-66.

21. Lytton SD, Ponto KA, Kanitz M, Matheis N, Kohn LD, Kahaly GJ. A novel thyroid stimulating immunoglobulin bioassay is a functional indicator of activity and severity of Graves' orbitopathy. J Clin Endocrinol Metab. 2010;95(5):2123-31.

22. Ponto KA, Kanitz M, Olivo PD, Pitz S, Pfeiffer N, Kahaly GJ. Clinical relevance of thyroid-stimulating immunoglobulins in graves' ophthalmopathy. Ophthalmology. 2011;118(11):2279-85.

23. Ponto KA, Diana T, Binder H, Matheis N, Pitz S, Pfeiffer N, et al. Thyroidstimulating immunoglobulins indicate the onset of dysthyroid optic neuropathy. J Endocrinol Invest. 2015;38(7):769-77.
24. Barrio-Barrio J, Sabater AL, Bonet-Farriol E, Velázquez-Villoria Á, Galofré JC. Graves' ophthalmopathy: VISA versus EUGOGO classification, assessment, and management. J Ophthalmol. 2015;2015:249125.

25. Kazim M, Gold KG. A review of surgical techniques to correct upper eyelid retraction associated with thyroid eye disease. Curr Opin Ophthalmol. 2011;22(5):391-3.

26. Marcocci C, Kahaly GJ, Krassas GE, Bartalena L, Prummel M, Stahl M, et al. Selenium and the course of mild Graves' orbitopathy. N Engl J Med. 2011;364(20):1920-31.

27. Zang S, Ponto KA, Kahaly GJ. Clinical review: intravenous glucocorticoids for Graves' orbitopathy: efficacy and morbidity. J Clin Endocrinol Metab. 2011;96(2):320-32.

28. Stiebel-Kalish H, Robenshtok E, Hasanreisoglu M, Ezrachi D, Shimon I, Leibovici L. Treatment modalities for Graves' ophthalmopathy: systematic review and metaanalysis. J Clin Endocrinol Metab. 2009;94(8):2708-16.

29. Marcocci C, Watt T, Altea MA, Rasmussen AK, Feldt-Rasmussen U, Orgiazzi J, et al. Fatal and non-fatal adverse events of glucocorticoid therapy for Graves' orbitopathy: a questionnaire survey among members of the European Thyroid Association. Eur J Endocrinol. 2012;166(2):247-53.

30. Bartalena L, Burch HB, Burman KD, Kahaly GJ. A 2013 European survey of clinical practice patterns in the management of Graves' disease. Clin Endocrinol (Oxf). 2016;84(1):115-20.

31. Mou P, Jiang LH, Zhang Y, Li YZ, Lou H, Zeng CC, et al. Common immunosup pressive monotherapy for graves' ophthalmopathy: a meta-Analysis. PLoS One. 2015;10(10):1-14.

32. Kahaly GJ, Pitz S, Hommel G, Dittmar M. Randomized, single blind trial of intravenous versus oral steroid monotherapy in Graves' orbitopathy. J Clin Endocrinol Metab. 2005;90(9):5234-40.

33. Zhu W, Ye L, Shen L, Jiao Q Huang $F$, Han $R$, et al. A prospective, randomized trial of intravenous glucocorticoids therapy with different protocols for patients with graves' ophthalmopathy. J Clin Endocrinol Metab. 2014;99(6):1999-2007

34. Bartalena L, Krassas GE, Wiersinga W, Marcocci C, Salvi M, Daumerie C, et al. Efficacy and safety of three different cumulative doses of intravenous methylprednisolone for moderate to severe and active Graves' orbitopathy. J Clin Endocrinol Metab. 2012;97(12):4454-63.

35. Vargas-Uricoechea H, Maya-Ruiz DE. Eficacia del tratamiento con esteroides para la oftalmopatía tiroidea. Rev Venez Endocrinol Metab. 2014:12(3):157-66.

36. Farach A, Carpenter LS. Radiation therapy for orbitopathy of thyroid eye disease. Int Ophthalmol Clin. 2016;56(1):81-93.

37. Sisti E, Menconi F, Leo M, Profilo MA, Mautone T, Mazzi B, et al. Long-term outcome of Graves' orbitopathy following high-dose intravenous glucocorticoids and orbital radiotherapy. J Endocrinol Invest. 2015;38(6):661-8.

38. Rajendram R, Bunce C, Lee RWJ, Morley AMS. Orbital radiotherapy for adult thyroid eye disease. Cochrane Database Syst Rev. 2012;(7):CD007114

39. Viani GA, Boin ACAC, De Fendi LI, Fonseca EC, Stefano EJ, Paula JS. Radiation therapy for graves' ophthalmopathy: a systematic review and meta-analysis of randomized controlled trials. Arq Bras Oftalmol. 2012;75(5):324-32.

40. Salvi M. Immunotherapy for Graves' ophthalmopathy. Curr Opin Endocrinol Diabetes Obes. 2014;21(5):409-14.

41. Perez-Moreiras JV, Gomez-Reino JJ, Maneiro JR, et al. Efficacy of Tocilizumab in Patients With Moderate-to-Severe Corticosteroid-Resistant Graves Orbitopathy: A Randomized Clinical Trial. Am J Ophthalmol. 2018;195:181-90.

42. Salvi M, Vannucchi G, Curro N, Campi I, Covelli D, Dazzi D, et al. Efficacy of B-cell targeted therapy with rituximab in patients with active moderate to severe Graves' orbitopathy: a randomized controlled study. J Clin Endocrinol Metab. 2015;100(2):422-31.

43. Stan MN, Garrity JA, Carranza Leon BG, Prabin T, Bradley EA, Bahn RS. Randomized controlled trial of rituximab in patients with Graves' orbitopathy. J Clin Endocrinol Metab. 2015;100(2):432-41.

44. Smith TJ, Kahaly GJ, Ezra DG, Fleming JC, Dailey RA, Tang RA, et al. Teprotumumab for thyroid-associated ophthalmopathy. N Engl J Med. 2017;376(18):1748-61.

45. Prummel MF, Mourits MP, Berghout A, Krenning EP, van der Gaag R, Koornneef $\mathrm{L}$, et al. Prednisone and cyclosporine in the treatment of severe Graves' ophthalmopathy. N Engl J Med. 1989;321(20):1353-9. 